\title{
Multivariant Non-Failure Analysis via Standard Abstract Interpretation
}

\author{
F. Bueno ${ }^{1}$, P. López-García ${ }^{1}$, and M. Hermenegildo ${ }^{1,2}$ \\ 1 School of Computer Science, Technical University of Madrid (UPM) \\ ${ }^{2}$ Depts. of Comp. Science and El. and Comp. Eng., U. of New Mexico (UNM) \\ \{bueno, pedro.lopez, herme\}@fi.upm.es \\ http://ww. cliplab.org/
}

\begin{abstract}
Non-failure analysis aims at inferring that predicate calls in a program will never fail. This type of information has many applications in functional/logic programming. It is essential for determining lower bounds on the computational cost of calls, useful in the context of program parallelization, instrumental in partial evaluation and other program transformations, and has also been used in query optimization. In this paper, we re-cast the non-failure analysis proposed by Debray et al. as an abstract interpretation, which not only allows to investigate it from a standard and well understood theoretical framework, but has also several practical advantages. It allows us to incorporate non-failure analysis into a standard, generic abstract interpretation engine. The analysis thus benefits from the fixpoint propagation algorithm, which leads to improved information propagation. Also, the analysis takes advantage of the multi-variance of the generic engine, so that it is now able to infer separate non-failure information for different call patterns. Moreover, the implementation is simpler, and allows to perform non-failure and covering analyses alongside other analyses, such as those for modes and types, in the same framework. Finally, besides the precision improvements and the additional simplicity, our implementation (in the Ciao/CiaoPP multiparadigm programming system) also shows better efficiency.
\end{abstract}

\section{Introduction}

Non-failure analysis involves detecting at compile time that, for any call belonging to a particular (possibly infinite) class of calls, a predicate will never fail. As an example, consider a predicate defined by the following two clauses:

$\operatorname{abs}(X, Y):-X>=0, Y$ is $X$.

$\operatorname{abs}(X, Y):-X<0, Y$ is $-X$.

and assume that we know that this predicate will always be called with its first argument bound to an integer, and the second argument a free variable. Obviously, for any particular call, one or the other of the tests $X>=0$ and $X<0$ may fail; however, taken together, one of them will always succeed. Thus, we can infer that calls to the predicate will never fail.

Being able to determine statically that a predicate will not fail has many applications. It is essential for determining lower bounds on the computational cost of goals since without such information a lower bound of almost zero (corresponding to an early failure) must often be assumed [10]. Detecting non-failure 
is also very useful in the context of parallelism because it allows avoiding unnecessary speculative parallelism and ensuring no-slowdown properties for the parallelized programs (in addition to using the lower bounds mentioned previously to perform granularity control) [11]. Non-failure information is also instrumental in partial evaluation and other program transformations, such as reordering of calls, and has also been used in query optimization in deductive databases [8]. It is also useful in program debugging, where it allows verifying user assertions regarding non-failure of predicates $[12,13]$. Finally, similar techniques can be used to detect the absence of errors or exceptions when running particular predicates.

A practical non-failure analysis has been proposed by Debray et al. [9]. In a similar way to the example above, this approach relies on first inferring mode and type information, and then testing that the constraints in the clauses of the predicate are entailed by the types of the input arguments, which is called a covering test. Covering cannot be inferred by examining the constraints of each clause separately: it is necessary to collect them together and examine the behavior of the predicate as a whole. Furthermore, non-failure of a given predicate depends on non-failure of other predicates being called and also possibly on the constraints in such predicates.

While [9] proposed the basic ideas behind non-failure analysis, only a simple, monovariant algorithm was proposed for propagating the non-failure information. In our experience since that proposal, we have found a need to improve it in several ways. First, information propagation needs to be improved, which leads us to a fixpoint propagation algorithm. Furthermore, the analysis really needs to be multi-variant, which means that it should be able to infer separate non-failure (and covering) information for different call patterns for a given predicate in a program. This is illustrated by the following example which, although simple, captures the very common case where the same (library) procedure is called from a program (in different points) for different purposes:

Example 1 Consider the (exported) predicate mv/3 (which uses the library predicate qsort $/ 2$ ), defined for the sake of discussion as follows:

$\operatorname{mv}(A, B, C):-q \operatorname{sort}(A, B), !, C=B$.

$\operatorname{mv}(A, B, C):-$ append $(A, B, D)$, qsort $(D, C)$.

Assume the following entry assertion for $\mathrm{mv} / 3$ :

:- entry mv(A,B,C) : (list (A, num), list (B, num), $\operatorname{var}(C))$.

which means that the predicate $\mathrm{mv}(\mathrm{A}, \mathrm{B}, \mathrm{C})$ will be called with $\mathrm{A}$ and $\mathrm{B}$ bound to lists of numbers, and $\mathrm{C}$ a free variable. A multi-variant non-failure analysis would infer two call patterns for predicate qsort $/ 2$ :

1. The call pattern qsort $(A, B)$ : (list $(A, n u m)$, list (B, num)), for which the analysis infers that it can fail and is not covered, and

2. the call pattern qsort $(A, B)$ : ( $\operatorname{list}(A, n u m), \operatorname{var}(B))$, for which the analysis infers that it will not fail and is covered.

This in turn allows the analysis to infer that the predicate mv $/ 3$ will not fail and is covered (for the call pattern expressed by the entry assertion).

However, a monovariant analysis only considers one call pattern per predicate. In particular, for predicate qsort $/ 2$, the call pattern used is qsort $(A, B)$ : 
(Iist (A,num), term(B) $)^{3}$ (which is the result of "collapsing" all call patterns which can appear in the program, so that precision is lost), for which it infers that qsort $/ 2$ can fail and is not covered. This causes the analysis to infer that the predicate mv $/ 3$ can fail (since the calls to qsort $/ 2$ in both clauses of predicate $\mathrm{mv} / 3$ are detected as failing) and is covered.

In order to address the different shortcomings of [9] in this paper we start by casting the ideas behind non-failure and covering analysis as an abstract interpretation [5]. This then allows us to incorporate non-failure analysis into a (somewhat modified) standard, generic abstract interpretation engine. This has several advantages. First of all, the analysis is now based on a standard and well studied theoretical framework. But, most importantly, being able to take advantage of standard and well developed analysis engines allows us to obtain a simpler and more efficient implementation, with better propagation of information, performing an efficient fixpoint. The non-failure and covering analyses can be performed alongside other abstract interpretation based analyses, such as those for modes and types, in the same framework. Furthermore, the analysis that we obtain is multi-variant (on calls and successes) thus inferring separate non-failure (and covering) information for different call patterns for a given predicate in a program. Finally, the abstract domain for non-failure can be easily enhanced to define a domain for determinacy of predicates.

Abstract Interpretation [5] is often proposed as a means for inferring properties of programs at compile-time. It was shown by Bruynooghe [2], Jones and Sondergaard [15], Debray [7], and Mellish [17] that this technique can be extended to flow analysis of programs in logic programming languages, and several frameworks or particular analyses have evolved since (e.g. [16, 20-22]). Abstract interpretation formalizes the relation between analysis and semantics, and, therefore, it is inherently semantics sensitive, different semantic definition styles yielding different approaches to program analysis. For logic programs we distinguish between two main approaches, namely bottom-up analysis and top-down analysis. We also distinguish between goal dependent and goal independent analyses. In this paper we use a goal dependent framework, since non-failure analysis is inherently goal dependent. In [3], Bruynooghe describes a framework for the goal-dependent, top-down abstract interpretation of logic programs. We use the PLAI/CiaoPP framework [12,13], which follows [3], but incorporates a number of optimizations and efficient fixpoint algorithms, described in $[18,19,14]$.

\section{Preliminaries}

We will denote $\mathcal{C}$ the universal set of constraints. We let $\theta \downarrow_{L}$ be the constraint $\theta$ restricted to the variables of the syntactic object $L$. We denote constraint entailment by $=$, so that $c_{1}=c_{2}$ denotes that $c_{1}$ entails $c_{2}$.

An atom has the form $p\left(t_{1}, \ldots, t_{n}\right)$ where $p$ is a predicate symbol and the $t_{i}$ are terms. A literal is either an atom or a constraint. A goal is a finite sequence of

\footnotetext{
${ }^{3}$ term(B) means that argument B can be bound to any term.
} 
literals. A rule is of the form $H:-B$ where $H$, the head, is an atom and $B$, the body, is a possibly empty finite sequence of literals. A constraint logic program, or program, is a finite set of rules. The definition of an atom $A$ in program $P$, def $n_{P}(A)$, is the set of variable renamings of rules in $P$ such that each renaming has $A$ as a head and has distinct new local (but not head) variables.

The operational semantics of a program is in terms of its "derivations" which are sequences of reductions between "states". A state $\langle G \mid \theta\rangle$ consists of a goal $G$ and a constraint store (or store for short) $\theta$. A state $\langle L:: G \mathbf{|} \theta\rangle$, where $L$ is a literal and :: denotes concatenation of sequences, can be reduced as follows:

1. If $L$ is a constraint and $\theta \wedge L$ is satisfiable, it is reduced to $\langle G \mid \theta \wedge L\rangle$.

2. If $L$ is an atom, it is reduced to $\langle B:: G \mid \theta\rangle$ for some rule $(L:-B) \in \operatorname{def} n_{P}(L)$. assuming for simplicity that the underlying constraint solver is complete. We use $S \leadsto P S^{\prime}$ to indicate that in program $P$ a reduction can be applied to state $S$ to obtain state $S^{\prime}$. Also, $S \sim_{P}^{*} S^{\prime}$ indicates that there is a sequence of reduction steps from state $S$ to state $S^{\prime}$. A derivation from state $S$ for program $P$ is a sequence of states $S_{0} \leadsto P S_{1} \leadsto P \ldots \leadsto P S_{n}$ where $S_{0}$ is $S$ and there is a reduction from each $S_{i}$ to $S_{i+1}$. Given a non-empty derivation $D$, we denote by curr_goal $(D)$ and curr_store $(D)$ the first goal and the store in the last state of $D$, respectively. E.g., if $D$ is the derivation $S_{0} \sim{ }_{P}^{*} S_{n}$ with $S_{n}=\langle g:: G \mid \theta\rangle$ then curr_goal $(D)=g$ and curr_store $(D)=\theta$. A query is a pair $(L, \theta)$ where $L$ is a literal and $\theta$ a store of an initial state $\langle L \mathbf{I} \theta\rangle$. The set of all derivations from $Q$ for $P$ is denoted derivations $(P, Q)$. We will denote sets of queries by $\mathcal{Q}$. We extend derivations to $\mathcal{Q}$ as follows: derivations $(P, \mathcal{Q})=\bigcup_{Q \in \mathcal{Q}} \operatorname{derivations}(P, Q)$.

The observational behavior of a program is given by its "answers" to queries. A finite derivation from a query $(L, \theta)$ for program $P$ is finished if the last state in the derivation cannot be reduced. A finished derivation from a query $(L, \theta)$ is successful if the last state is of the form $\left\langle\right.$ nil $\left.\mathbf{|} \theta^{\prime}\right\rangle$, where nil denotes the empty sequence. The constraint $\theta^{\prime} \downarrow_{L}$ is an answer to $(L, \theta)$. We denote by answers $(P, Q)$ the set of answers to query $Q$. A finished derivation is failed if the last state is not of the form $\langle n i l| \overrightarrow{\mid} \theta$. Note that derivations $(P, \mathcal{Q})$ contains not only finished derivations but also all intermediate derivations. A query $Q$ finitely fails in $P$ if derivations $(P, Q)$ is finite and contains no successful derivation.

Abstract Interpretation. Abstract interpretation [5] is a technique for static program analysis in which execution of the program is simulated on an abstract domain $\left(D_{\alpha}\right)$ which is simpler than the actual, concrete domain $(D)$. For this study, we restrict to complete lattices over sets both for the concrete $\left\langle 2^{D}, \subseteq\right\rangle$ and abstract $\left\langle D_{\alpha}, \sqsubseteq\right\rangle$ domains.

Abstract values and sets of concrete values are related via a pair of monotonic mappings $\langle\alpha, \gamma\rangle$ : abstraction $\alpha: 2^{D} \rightarrow D_{\alpha}$, and concretization $\gamma: D_{\alpha} \rightarrow 2^{D}$, such that $\forall x \in 2^{D}: \gamma(\alpha(x)) \supseteq x$ and $\forall y \in D_{\alpha}: \alpha(\gamma(y))=y$. In general $\sqsubseteq$ is defined so that the operations of least upper bound $(\sqcup)$ and greatest lower bound ( $\sqcap$ mimic those of $2^{D}$ in a precise sense:

$$
\begin{gathered}
\forall \lambda, \lambda^{\prime} \in D_{\alpha}: \lambda \sqsubseteq \lambda^{\prime} \Leftrightarrow \gamma(\lambda) \subseteq \gamma\left(\lambda^{\prime}\right) \\
\forall \lambda_{1}, \lambda_{2}, \lambda^{\prime} \in D_{\alpha}: \lambda_{1} \sqcup \lambda_{2}=\lambda^{\prime} \Leftrightarrow \gamma\left(\lambda_{1}\right) \cup \gamma\left(\lambda_{2}\right)=\gamma\left(\lambda^{\prime}\right)
\end{gathered}
$$$$
\forall \lambda_{1}, \lambda_{2}, \lambda^{\prime} \in D_{\alpha}: \lambda_{1} \sqcap \lambda_{2}=\lambda^{\prime} \Leftrightarrow \gamma\left(\lambda_{1}\right) \cap \gamma\left(\lambda_{2}\right)=\gamma\left(\lambda^{\prime}\right)
$$ 
Goal dependent abstract interpretation takes as input a program $P$, an abstract domain $D_{\alpha}$, and a description $\mathcal{Q}_{\alpha}$ of the possible initial queries to $P$, given as a set of abstract queries. An abstract query is a pair $(L, \lambda)$, where $L$ is an atom (for one of the exported predicates) and $\lambda \in D_{\alpha}$ describes the initial stores for $L$. A set $\mathcal{Q}_{\alpha}$ represents the set of queries $\gamma\left(\mathcal{Q}_{\alpha}\right)$, which is defined as $\gamma\left(\mathcal{Q}_{\alpha}\right)=$ $\left\{(L, \theta) \mid(L, \lambda) \in \mathcal{Q}_{\alpha} \wedge \theta \in \gamma(\lambda)\right\}$. Such an abstract interpretation computes a set of triples Analysis $\left(P, \mathcal{Q}_{\alpha}, D_{\alpha}\right)=\left\{\left\langle L_{p}, \lambda^{c}, \lambda^{s}\right\rangle \mid p\right.$ is a predicate of $\left.P\right\}$, where $L_{p}$ is a (program) atom for predicate $p$. Note that, the analysis being multivariant (on calls), it may compute several tuples of the form $\left\langle L_{p}, \lambda^{c}, \lambda^{s}\right\rangle$ for different call patterns $\left\langle L_{p}, \lambda^{c}\right\rangle$ of each predicate $p$ (including different program atoms $L_{p}$ ). If $p$ is detected to be dead code then $\lambda^{c}=\lambda^{s}=\perp$. As usual in abstract interpretation, $\perp$ denotes the abstract constraint such that $\gamma(\perp)=\emptyset$, whereas $T$ denotes the most general abstract constraint, i.e., $\gamma(T)=D$.

\section{The Abstract Interpretation Framework}

PLAI is an analysis system based on the abstract interpretation framework of Bruynooghe [3] with the optimizations described in [18]. The framework works on an abstraction of the (SLD) AND-OR trees of the execution of a program for given entry points. The abstract AND-OR graph makes it possible to provide information at each program point, a feature which is crucial for many applications (such as, e.g., reordering, automatic parallelization, or garbage collection).

Program points and abstract substitutions are related as follows. Consider a clause $h:-p_{1}, \ldots, p_{n}$. Let $\lambda_{i}$ and $\lambda_{i+1}$ be the abstract substitutions to the left and right of the subgoal $p_{i}, 1 \leq i \leq n$ in this clause. Then $\lambda_{i}$ and $\lambda_{i+1}$ are, respectively, the abstract call substitution and the abstract success substitution for the subgoal $p_{i}$. For this same clause, $\lambda_{1}$ is the abstract entry substitution and $\lambda_{n+1}$ is the abstract exit substitution. Entry and exit substitutions are denoted respectively $\beta_{\text {entry }}$ and $\beta_{\text {exit }}$ when projected on the variables of the clause head.

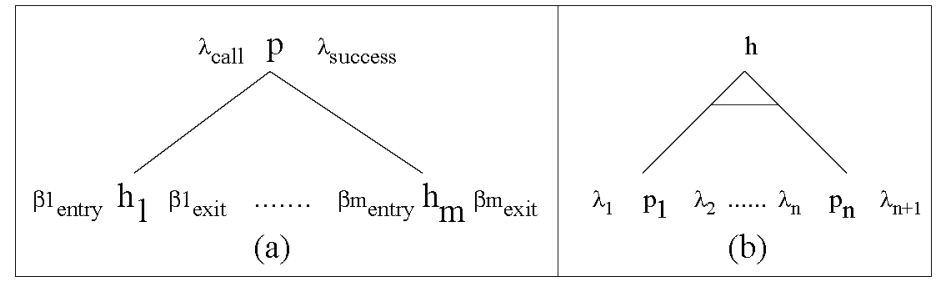

Fig. 1. Illustration of the Top-Down Abstract Interpretation Process

Computing the success substitution from the call substitution is done as follows (see Figure 1(a)). Given a call substitution $\lambda_{\text {call }}$ for a subgoal $p$, let $h_{1}, \ldots, h_{m}$ be the heads of clauses which unify with $p$. Compute the entry substitutions $\beta 1_{\text {entry }}, \ldots, \beta m_{\text {entry }}$ for these clauses. Compute their exit substitutions $\beta 1_{\text {exit }}, \ldots, \beta m_{\text {exit }}$ as explained below. Compute the success substitutions $\lambda 1_{\text {success }}, \ldots, \lambda m_{\text {success }}$ from the corresponding exit substitutions. At this point, 
all different success substitutions can be considered for the rest of the analysis, or a single success substitution $\lambda_{\text {success }}$ for subgoal $p$ computed by means of an aggregation operation for $\lambda 1_{\text {success }}, \ldots, \lambda m_{\text {success }}$. This aggregator is usually the LUB (least upper bound) of the abstract domain. In the first case the analysis is multi-variant on successes, in the second case it is not.

Computing the exit substitution from the entry substitution is straightforward (see Figure 1(b)). Given a clause $h:-p_{1}, \ldots, p_{n}$ and an entry substitution $\beta_{\text {entry }}$ for the clause head $h, \lambda_{1}$ is the call substitution for $p_{1}$. This one is computed simply by adding to $\beta_{\text {entry }}$ an abstraction for the free variables in the clause. The success substitution $\lambda_{2}$ for $p_{1}$ is computed as explained above (essentially, by repeating this same process for the clauses which match $p_{1}$ ). Similarly, $\lambda_{3}, \ldots, \lambda_{n+1}$ are computed. The exit substitution $B_{e x i t}$ for this clause is precisely the projection onto $h$ of $\lambda_{n+1}$.

If, from a different subgoal in the program, a different entry substitution is computed for an already analyzed clause, different call substitutions will appear (for $p_{1}$ and possibly the other subgoals). These substitutions can be collapsed using the LUB operation, or a different node in the graph can be computed. In the latter solution, different nodes exist in the graph for each call substitution and subgoal, thus yielding an analysis which is multi-variant on calls.

Note that the framework itself is domain independent. To instantiate it, a particular analysis needs to define an abstract domain and abstract unification, and the $\sqsubseteq$ relation, which in turn defines $\sqcup$ (LUB). Abstract unification is divided into two in the framework, so that it is required to define: (1) how to compute the entry substitution for a clause $C$ given a subgoal $p$ (which unifies with the head of $C$ ) and its call substitution; and (2) how to compute the success substitution for a subgoal $p$ given its call substitution and the exit substitution for a clause $C$ whose head unifies with $p$. We formalize this with functions entry_to_exit and call_to_success in Figure 2. The domain dependent functions used there are:

- call_to_entry $(p(\bar{u}), C, \lambda)$ which gives an abstract substitution describing the effects on $\operatorname{vars}(C)$ of unifying $p(\bar{u})$ with head $(C)$ given an abstract substitution $\lambda$ describing $\bar{u}$

- exit_to_success $(\lambda, p(\bar{u}), C, \beta)$ which gives an abstract substitution describing $\bar{u}$ accordingly to $\beta$ (which describes vars $($ head $(C)$ )) and the effects of unifying $p(\bar{u})$ with head $(C)$ under the abstract substitution $\lambda$ describing $\bar{u}$,

- extend $\left(\lambda, \lambda^{\prime}\right)$ which extends abstract substitution $\lambda$ to incorporate the information in $\lambda^{\prime}$ in a way that it is still consistent,

- project_in $(\bar{v}, \lambda)$ which extends $\lambda$ so that it refers to all of the variables $\bar{v}$,

- project_out $(\bar{v}, \lambda)$ which restricts $\lambda$ to only the variables $\bar{v}$.

In the presence of recursive predicates, analysis requires a fixpoint computation. In $[18,19]$ a fixpoint algorithm was proposed for the framework that localizes fixpoint computations to only the strongly connected components of (mutually) recursive predicates. Additionally, an initial approximation to the fixpoint is computed from the non-recursive clauses of the recursive predicate. Fixpoint convergence is accelerated by updating this value with the information from every clause analyzed in turn. The algorithm is (schematically) shown in Figure 3. For a complete description see $[18,19]$. 


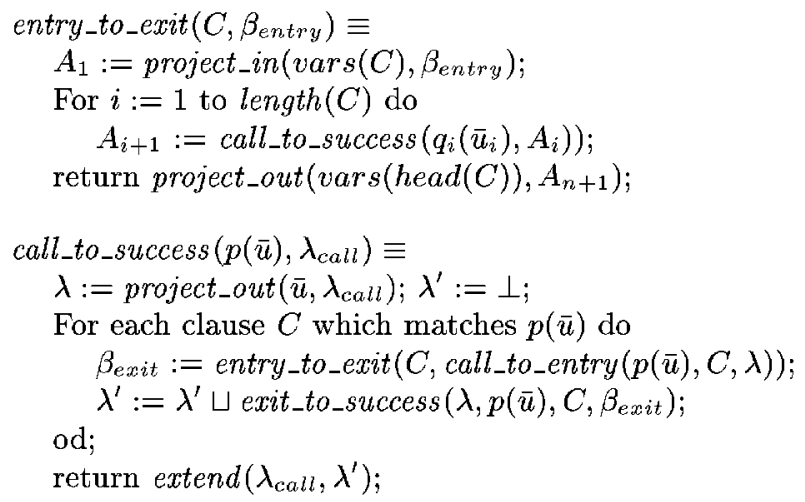

Fig. 2. The Top-Down Framework

\section{Abstract Framework, Domain, and Operations for Non-Failure Analysis}

In the non-failure analysis, the covering test is instrumental. In fact, covering can be seen as a notion that characterizes the fact that execution of a query will not finitely fail, i.e., if it has finished derivations then at least one is successful. Note that, as in [9], non-failure does not imply success: a predicate that is non-failing may nevertheless not produce an answer because it does not terminate.

Definition 1 (Covering). Given computation state $\langle g:: G \mathbf{|} \theta\rangle$ in the execution of program $P$, define the global answer constraint of goal $g$ in store $\theta$ as:

$c=\vee\left\{\right.$ curr_store $\left(D_{i}^{\prime}\right) \mid D_{i}^{\prime} \in$ derivations $(P,\langle g, \theta\rangle)$ and is maximal $\}$

Let $\bar{u}$ denote the variables of $g$ already constrained in $\theta$, call them the input variables. We say that $g$ is covered in $\theta$ iff $\theta \downarrow_{\bar{u}} \models c \downarrow_{\bar{u}}$.

It is not difficult to show that, in a pure language, where failure can only be caused by constraint store inconsistency, covering is a sufficient condition for non-failure. Indeed, if $g$ is covered in $\theta$, i.e., $\theta \downarrow_{\bar{u}}=c \downarrow_{\bar{u}}$, then one of the disjunctions in (the projection of) $c$ is entailed. This corresponds to a (maximal) derivation of $\langle g, \theta\rangle$, and this derivation cannot be failed, since, if it were, it would be inconsistent, and no inconsistent constraint can be entailed by a consistent one. Therefore, either such derivation is infinite, or, if finite, it is successful. Thus:

If $g$ is covered in $\theta$ then $\langle g, \theta\rangle$ does not finitely fail.

A key issue in non-failure analysis will thus be how to approximate the current store and the global answer constraint so that covering can be effectively and accurately approximated. In [9] such an approximation is defined in the following terms: A goal is non-failing if there is a subset of clauses of the predicate which do not fail and which match the input types of the goal. This "matching" is the so-called covering test, and basically amounts to the analysis being able to gather, for each such clause, enough constraints on the input variables of the 


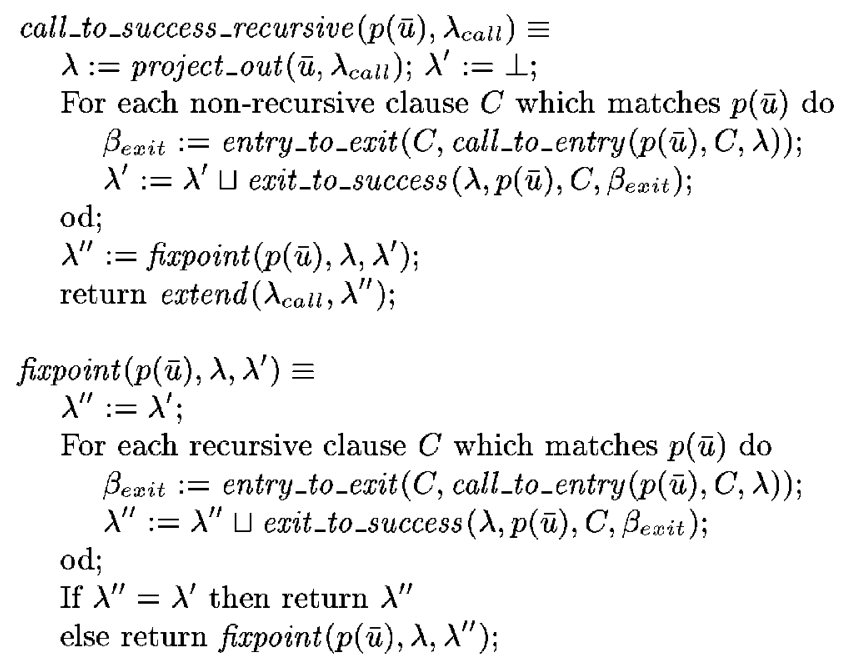

Fig. 3. The Fixpoint Computation

goal to be able to prove that, for each of the variables, any element in the corresponding type satisfies at least the constraint gathered for one clause. An analysis for non-failure thus needs to traverse the clauses of a predicate to check non-failure of the clause body goals, collect constraints that approximate the global answer constraint, and finally check that they cover the input types of the original goal. In the rest of this section, we show how to accommodate the abstract interpretation based framework of the previous section to perform these tasks, and define an abstract domain suitable for them.

\subsection{Abstract Domain}

The abstractions for non-failure analysis are made of four components. The first two are (abstractions of) constraints that represent the current store and the global answer constraint for the current goal. This is the core part of the domain. The other two components carry the results of the covering test, specifying if the current constraint store covers the global answer constraint, and if this implies that the computation may fail or not. The covering and non-failure information is represented by values of the set $\mathcal{B}=\{T, \overline{0}, \overline{1}, \perp\}$, where $\overline{0}$ and $\overline{1}$ are not comparable in the ordering. For covering, $\overline{0}$ is interpreted as "not covered" and $\overline{1}$ as covered. For non-failure, $\overline{0}$ is interpreted as "not failing" and $\overline{1}$ as failing.

Definition 2 (Abstract Domain). Let $\mathcal{C}^{\alpha_{1}}$ and $\mathcal{C}^{\alpha_{2}}$ be abstract domains for $\mathcal{C}$. The abstract domain for non-failure is the set

$$
\mathcal{F}=\left\{(s, c, o, f) \mid s \in \mathcal{C}^{\alpha_{1}}, c \in \mathcal{C}^{\alpha_{2}}, o \in \mathcal{B}, f \in \mathcal{B}\right\}
$$

The ordering in domain $\mathcal{F}$ is induced from that in $\mathcal{B}$, so that (overloading $\sqsubseteq$ ):

$$
\left(s_{1}, c_{1}, o_{1}, f_{1}\right) \sqsubseteq\left(s_{2}, c_{2}, o_{2}, f_{2}\right) \text { iff } f_{1} \sqsubseteq f_{2}
$$


In an element $(s, c, o, f) \in \mathcal{F}$, components $s$ and $c$ are abstractions $\alpha_{1}$ and $\alpha_{2}$ of the constraint domain $\mathcal{C}$. The usual approximations used (e.g., in [9]) are types (and modes) for $s$, and a finite set of (concrete) constraints for $c$.

Definition 3 (Abstraction Function). The abstraction of a derivation $D$ in the execution of program $P$, such that curr_store $(D)=\theta$ and curr_goal $(D)=g$, and the input variables and global answer constraint of $g$ in $\theta$ are respectively $\bar{u}$ and $c$, is $\alpha(D)=\left(\theta^{\alpha_{1}}, c^{\alpha_{2}}, o, f\right)$, where:

$$
f=\left\{\begin{array}{ll}
\overline{1} & \text { if } D \text { is failed } \\
\overline{0} & \text { otherwise }
\end{array} \text { and } o= \begin{cases}\overline{1} & \text { if } \theta^{\alpha_{1}} \downarrow_{\bar{u}} \models^{\alpha} c^{\alpha_{2}} \downarrow_{\bar{u}} \\
\overline{0} & \text { otherwise }\end{cases}\right.
$$

It is easy to show that such an abstraction is correct, provided that $\alpha_{1}$ and $\alpha_{2}$ are also correct abstractions, and that the corresponding abstract covering test $\left(=^{\alpha}\right)$ correctly approximates Definition 1 . For $\alpha_{1}$ we have already mentioned the use of type and mode information. One possibility for $\alpha_{2}$ is to use only those constraints appearing explicitly in the clause bodies of the predicate whose covering test is to be performed (the current goal $g$ in the derivation).

Example 2 Consider the following (contrived) predicates:

$\mathrm{P}(\mathrm{X}, \mathrm{Y}, \mathrm{Z}):-\mathrm{X}=<\mathrm{Y}, \mathrm{q}(\mathrm{X}, \mathrm{Z})$.

$\mathrm{q}(\mathrm{X}, \mathrm{Y}):-\mathrm{X}=<\mathrm{Y}$.

The global answer constraint for $\mathrm{p}(\mathrm{X}, \mathrm{Y}, \mathrm{Z})$ is $\mathrm{X}=<\mathrm{Y} \wedge \mathrm{X}=<\mathrm{Z}$, but it can be approximated simply by $\mathrm{X}=<\mathrm{Y}$, the only constraint in the definition of $\mathrm{P} / 3$.

One rationale for the above choice might be that collecting all constraints in derivations may not be possible during a compile-time analysis (since such constraints are only known during execution), or may lead to non-termination of the analysis. However, the first problem can be alleviated by proper abstractions of the tests (such as a depth-k abstraction, in a way similar to [6]), and the second problem only occurs for recursive predicates. Thus, the most simple solution to the termination problem is to avoid collecting constraints in recursive calls. ${ }^{4}$

Example 3 The global answer constraint for the predicate sorted/1 defined below includes a constraint for each two elements in the input list, the length of which is not in general known at compile-time.

sorted ( []).

$\operatorname{sorted}\left(\left[{ }_{-}\right]\right)$.

$\operatorname{sorted}([\mathrm{X}, \mathrm{Y} \mid \mathrm{L}]):-\mathrm{X}=<\mathrm{Y}, \operatorname{sorted}([\mathrm{Y} \mid \mathrm{L}])$.

Our solution to this problem ${ }^{5}$ is to collect only constraints that refer literally to the predicate arguments in the program clause head, which also excludes in general (but not always) the constraints arising from recursive calls.

\footnotetext{
${ }^{4}$ Note that this does not imply that recursive calls are simply ignored. They need to be considered to check that they are indeed non-failing, even though their global answer constraint is not computed.

${ }^{5}$ However, we plan to investigate other solutions. In particular, the use of a depth-k abstraction seems to be a very promising one.
} 
Example 4 Consider again the predicate sorted/1 defined in the previous example. We collect constraints only for the clause head argument $[X, Y \mid L]$, which amounts to only one constraint: $\mathrm{X}=<\mathrm{Y}$ (since the recursive call does not provide constraints for the head arguments that appear literally in the program).

Consider, on the other hand, predicate $\mathrm{p} / 3$ of Example 2. In this case the complete global answer constraint for $\mathrm{P}(\mathrm{X}, \mathrm{Y}, \mathrm{Z})$ will be collected: $\mathrm{X}=<\mathrm{Y} \wedge \mathrm{X}=<\mathrm{Z}$, since the two single constraints can be "projected" onto the clause head.

Note that such a solution yields an under-approximation of the global answer constraints. Given the use of type and mode information, which are in general over-approximations, we have that, for any element $(s, c, o, f) \in \mathcal{F}$, given current constraint store $\theta$ and global answer constraint $\omega, s=\theta^{\alpha_{1}}$ is an overapproximation of $\theta$, and $c=\omega^{\alpha_{2}}$ is an under-approximation of $\omega$. In this situation, it is not difficult to prove that $\theta^{\alpha_{1}} \downarrow_{\bar{u}} \models^{\alpha} \omega^{\alpha_{2}} \downarrow_{\bar{u}}$ correctly approximates covering: $\theta \downarrow_{\bar{u}} \models \omega \downarrow_{\bar{u}}$.

\subsection{Abstract Operations}

Abstract values $(s, c, o, f) \in \mathcal{F}$ are built during analysis in the following way: $f$ is carried along during the abstract computation by the abstract operations below, $o$ is computed from the covering test, $c$ is collected as explained above, and for $s$, type and mode analysis is performed. Thus, our analysis is in fact three-fold: it carries on mode, type, and non-failure analyses simultaneously. We focus now on the abstract operations for non-failure, given that those for types and modes are standard:

- call_to_entry $(p(\bar{u}), C, \lambda)$ solves head unification $p(\bar{u})=$ head $(C)$, and checks that it is consistent with the $c$ component of $\lambda$. If it is not, it returns $\perp$, otherwise, the resulting abstraction.

If $p(\bar{u}) \in \mathcal{C}$, i.e., if it happens to be a constraint itself, then no clause $C$ exists, and $p(\bar{u})$ itself is added to the $c$ component. In this case the following exit_to_success function is not called.

- exit_to_success $(\lambda, p(\bar{u}), C, \beta)$ adds the equations resulting from unification $p(\bar{u})=h e a d(C)$ to the $c$ component of $\beta$ and projects it onto vars $(\bar{u})$.

It is the projection performed here that gets rid of useless constraints, like in the case of Example 4. Constraints that cannot be projected onto the (goal) variables $\bar{u}$ are simply dropped in the analysis.

- $\lambda \uplus \lambda^{\prime}$ adds abstraction $\lambda$ to the set $\lambda^{\prime}$ if $\lambda$ is non-failing.

- extend $\left(\lambda, \lambda^{\prime}\right)$ performs the covering test for $\lambda^{\prime}$ (a set of abstractions); if it is successful, the $c$ component of $\lambda^{\prime}$ is merged with that of $\lambda$.

This operation uses the covering algorithm described in [9], which takes the global answer constraint $c$ and a type assignment for the input variables appearing in $c$. Given a finite set of variables $V$, a type assignment over $V$ is a mapping from $V$ to a set of types. This is computed from the type information in the first component of $\lambda$. Input variables are determined from the mode information in that same component. The global answer constraint is obtained as the disjunction of the $c$ components of each abstraction in $\lambda^{\prime}$. 


\subsection{Adapting the Analysis Framework}

The framework described in the previous section is not adequate for non-failure analysis. The main reason for this is that the aggregation function for the successive exit abstractions of the different clauses is not the LUB anymore. In nonfailure analysis, the constraints for each clause need to be gathered together, and a covering test on the set of constraints needs to be performed. Another difference is that the covering test should only consider constraints from clauses that are not guaranteed to fail altogether; ${ }^{6}$ therefore the aggregator must be able to discriminate abstract substitutions on this criterion.

We have adapted the definition of the call_to_success function to reflect the aggregation operator. The adapted definition is shown in Figure 4. Note that, as a result of this, $\lambda^{\prime}$ in the algorithm is not anymore an abstract substitution, but a set of them. This is input to extend, which is in charge of the covering test.

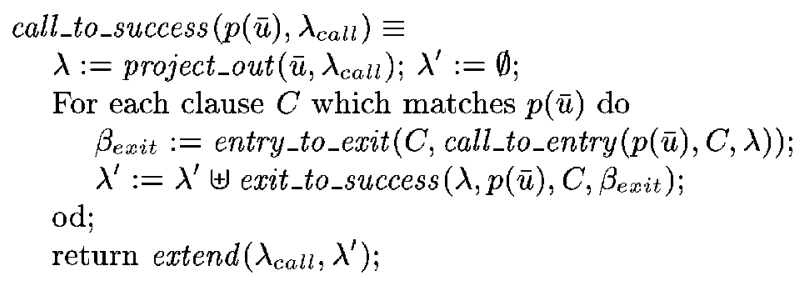

Fig. 4. The Top-Down Framework for Non-Failure Analysis

When fixpoint computation is required, adapting the framework is a bit more involved. Basically, since the aggregation operator is not LUB, fixpoint detection cannot be performed right after the success substitution has been computed. Normally, it is the LUB that is used for updating the successive approximations to the fixpoint value, and fixpoint detection works by simply comparing the initial and the final values for the success substitution. In non-failure analysis, the covering test must be performed first, and only after this one has been performed, the test for the fixpoint can be done. The resulting algorithm is shown in Figure 5. It is basically a simpler fixpoint iterator over the function call_to_success abandoning the sophisticated fixpoint computation of Figure 3.

A Running Example We now illustrate our analysis by means of a detailed example on how it will proceed. Consider the program (fragment) below:

qsort (As, Bs):- qsort (As, Bs, []).

qsort ( $[X \mid L], R, R 2)$ :-

partition(L, X,L1,L2), qsort (L2,R1,R2), qsort (L1, R, [X|R1]).

qsort ( []$, R, R)$.

partition ( []$,-,[],[])$.

partition([E|R], C, [E|Left1], Right):- E < C, partition(R,C,Left1,Right).

partition([E|R],C,Left, [E|Right1]):- E >= C, partition(R,C,Left,Right1).

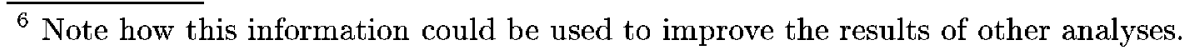




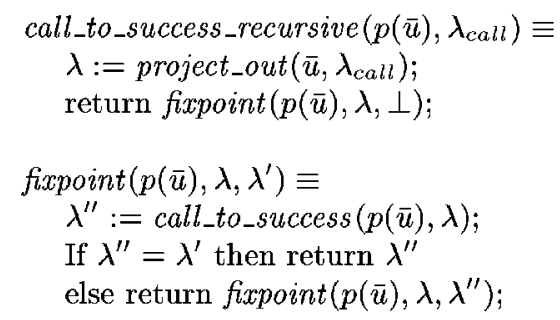

Fig. 5. The Fixpoint Computation for Non-Failure Analysis

Let the abstract call pattern for atom qsort (As, Bs, [] ) be $(\{\operatorname{list}(A s, n u m), \operatorname{var}(B s)\}, \operatorname{true}, \overline{1}, \overline{0})$.

Upon entering the first clause defining qsort/3, the result of call_to_entry (restricted to the head variables) is

$$
(\{\operatorname{num}(X), \operatorname{list}(L, \operatorname{num}), \operatorname{var}(R),[](R 2)\}, \operatorname{true}, \overline{1}, \overline{0})^{7}
$$

plus, additionally, $\{\operatorname{var}(R 1), \operatorname{var}(L 1), \operatorname{var}(L 2)\}$ for the free variables in the clause. Once projected, this gives the call pattern for the first literal in that clause:

$$
(\{\operatorname{list}(L, \operatorname{num}), \operatorname{num}(X), \operatorname{var}(L 1), \operatorname{var}(L 2)\}, \operatorname{true}, \overline{1}, \overline{0}) \text {. }
$$

We omit the analysis of the partition predicate. After the fixpoint computation for this predicate, however, we will have a set of three abstract elements corresponding to the abstraction of the three clauses. For brevity, we express such set as a single abstraction where it is the $c$ component that is a set, instead. ${ }^{8}$ Note that this is possible because all other components (types, modes, covering, non-failure) of the abstractions in the set are the same. Thus, we have:

$$
\begin{aligned}
& (\{\operatorname{list}(L, \operatorname{num}), \operatorname{num}(X), \operatorname{list}(L 1, \operatorname{num}), \operatorname{list}(L 2, \operatorname{num})\}, \\
& \left\{L=[] \wedge L 1=[] \wedge L 2=[], \quad L=\left[\left.E\right|_{-}\right] \wedge E<X \wedge L 1=\left[\left.E\right|_{-}\right],\right. \\
& \left.L=\left[\left.E\right|_{-}\right] \wedge E>=X \wedge L 2=\left[\left.E\right|_{-}\right]\right\},
\end{aligned}
$$

This is now extended (by abstract function extend) to the corresponding program point of the clause of qsort. First, the covering test is performed, and it succeeds, since list $(L, n u m), \operatorname{num}(X)$ covers indeed the global answer constraint projected onto the input variables:

$$
\left(L=\left[\left.E\right|_{-}\right] \wedge(E<X \vee E>=X)\right) \vee L=[.
$$

Therefore, computation is still covered and non-failing. This, together with the projection of the $c$ component onto the variables of the first clause of qsort, yields success abstraction for partition:

$$
\begin{aligned}
& (\{\operatorname{num}(X), \operatorname{list}(L, \operatorname{num}), \operatorname{var}(R), \operatorname{var}(R 1),[](R 2), \\
& \quad \operatorname{list}(L 1, \operatorname{num}), \operatorname{list}(L 2, \operatorname{num})\}, \\
& \text { true, }, \overline{1}, \overline{0})
\end{aligned}
$$

where the $c$ component is still true since the projection onto the clause variables factors out the previously computed global answer constraint. Now, analysis will proceed into call qsort (L2, R1,R2) with

$$
(\{\operatorname{list}(L 2, \operatorname{num}), \operatorname{var}(R 1),[](R 2)\}, \text { true }, \overline{1}, \overline{0}) \text {. }
$$

\footnotetext{
${ }^{7}$ To be concise, we denote with [](A) that the type of $A$ is that of the empty lists.

${ }^{8}$ This very same "trick" is used in the implementation.
} 
Since this is basically the same call pattern that we started with, no new fixpoint computation is started in this case. ${ }^{9}$ On the other hand, a new fixpoint computation is started for the second recursive call qsort $(\mathrm{L} 1, \mathrm{R},[\mathrm{X} \mid \mathrm{R} 1])$ with

$(\{l i s t(L 1, n u m), \operatorname{var}(R), \operatorname{num}(X), \operatorname{list}(R 1$, num $)\}$, true $, \overline{1}, \overline{0})$.

This is a new call pattern for the qsort predicate, which initiates a new fixpoint computation. The fixpoint value obtained in this computation is the same abstraction, except for the type of $R$ which on output is a list. Finally, exit_to_success now lifts this result to the original goal qsort (As, Bs, []) giving: $(\{\operatorname{list}($ As, num $), \operatorname{list}(B s$, num $)\}$, As $=[-\mid-], \overline{1}, \overline{0})$.

The analysis of the non-recursive clause immediately gives:

$$
(\{[](A s),[](B s)\}, A s=[] \wedge B s=[], \overline{1}, \overline{0}),
$$

and extend computes the covering test for the set of the above two abstractions with the initial input abstraction, in which the input types are $l i s t(A s, n u m)$. Certainly, this type covers the (projected) global answer constraint $A s=[-\mid-] \mathrm{V}$ $A s=[]$. Thus, the goal is still covered and non-failing.

Finally, since the abstraction now computed is only the result of a first iteration of the fixpoint computation, a new iteration is started. The result in this case is the same, and fixpoint computation finishes with that very same result.

\section{$5 \quad$ Implementation Results}

We have constructed a prototype implementation in (Ciao) Prolog by adapting the framework of the PLAI implementation and defining the abstract operations for non-failure analysis that we have described in this paper. Most of these abstract operations have been implemented by reusing code of the implementation in [9], such as for example, the covering algorithm. We have incorporated the prototype in the Ciao/CiaoPP multiparadigm programming system $[12,13,4]$ and tested it on the benchmarks used in the non-failure analysis of Debray $e t$ al. [9], plus some benchmarks exhibiting paradigmatic behaviours, plus a last group with those used in the cardinality analysis of Braem et al. [1]. These two analyses are the closest related previous work that we are aware of. Some relevant results of these tests for non-failure analysis are presented in Table 1. Program lists the program names, $\mathbf{N}$ the number of predicates in the program, $\mathbf{F}$ and $\mathbf{C}$ are the number of non-failing predicates detected by the non-failure analysis in [9], and the cardinality analysis in [1], respectively.

Note that our multi-variant analysis can infer several variants (call patterns) for the same predicate, where some of them may be non-failing (resp. covered) and the other ones can be failing (resp. not covered). For instance, in the case of the program $M v$ in Table 1 (also described in Example 1), which has 4 predicates (mv $/ 3$, qsort $/ 2$, partition $/ 4$ and append $/ 3$ ), the analysis infers one variant for $\mathrm{mv} / 3$, which is non-failing and covered, 2 variants for qsort $/ 2$ (one of them which is non-failing and covered, and the other one which is failing and not covered), one variant for partition/4, which is non-failing and covered, and 3 variants

\footnotetext{
${ }^{9}$ Here, we save the reader from some more fixpoint iterations that will be taking place. However, the results are as indicated.
} 
for append $/ 3$ ( 2 of them which are non-failing and covered, and the other one which is failing and not covered). For this reason, and in order to make the results comparable, column AF shows two figures (both corresponding to the analysis presented in this paper): the number of predicates such that all of their variants (call patterns) are detected as non-failing, and (between parenthesis) the number of predicates such that some of their variants are detected as non-failing (this second figure is omitted if it is equal to the first one).

\begin{tabular}{|l|r|r|r|r|r|r|r|r|r|}
\hline \hline Program & $\mathbf{N}$ & $\mathbf{A F}$ & $\mathbf{F}$ & $\mathbf{C}$ & $\mathbf{A C o v}$ & $\mathbf{C o v}$ & $\mathbf{T}_{A F}$ & $\mathbf{T}_{F}$ & $\frac{T_{A F}}{T_{F}}$ \\
\hline \hline Hanoi & 2 & 2 & 2 & N/A & 2 & 2 & 33 & 242 & $\mathbf{0 . 1 4}$ \\
\hline Fib & 1 & 1 & 1 & N/A & 1 & 1 & 17 & 22 & $\mathbf{0 . 7 7}$ \\
\hline Tak & 1 & 1 & 1 & N/A & 1 & 1 & 9 & 11 & $\mathbf{0 . 8 2}$ \\
\hline Subs & 1 & 1 & 1 & N/A & 1 & 1 & 5 & 33 & $\mathbf{0 . 1 5}$ \\
\hline Reverse & 2 & 2 & 2 & N/A & 2 & 2 & 17 & 29 & $\mathbf{0 . 5 9}$ \\
\hline Mv & 4 & $2(4)$ & 1 & N/A & $2(4)$ & 2 & 54 & 102 & $\mathbf{0 . 5 3}$ \\
\hline Zebra & 6 & 2 & 1 & N/A & $5(6)$ & 4 & 1008 & 1100 & $\mathbf{0 . 9 2}$ \\
\hline Family & 3 & 3 & 1 & N/A & 3 & 2 & 10 & 18 & $\mathbf{0 . 5 6}$ \\
\hline Blocks & 7 & $1(2)$ & 0 & N/A & $\mathbf{4}(5)$ & 4 & 30 & 59 & $\mathbf{0 . 5 1}$ \\
\hline Reach & 2 & 2 & 0 & N/A & 2 & 1 & 19 & 30 & $\mathbf{0 . 6 3}$ \\
\hline Bid & 20 & $5(8)$ & 5 & N/A & $14(17)$ & 14 & 3089 & 3369 & $\mathbf{0 . 9 2}$ \\
\hline Occur & 4 & $1(3)$ & 1 & N/A & $1(3)$ & 1 & 69 & 78 & $\mathbf{0 . 8 8}$ \\
\hline \hline Plan & 16 & $5(8)$ & 3 & 0 & $11(13)$ & 10 & 2626 & 4128 & $\mathbf{0 . 6 4}$ \\
\hline Qsort & 3 & 3 & 3 & 0 & 3 & 3 & 29 & 65 & $\mathbf{0 . 4 5}$ \\
\hline Qsort2 & 5 & 3 & 3 & 0 & 3 & 3 & 33 & 76 & $\mathbf{0 . 4 3}$ \\
\hline Queens & 5 & $2(3)$ & 2 & 0 & $3(\mathbf{4})$ & 3 & 60 & 74 & $\mathbf{0 . 8 1}$ \\
\hline Pg & 10 & $2(3)$ & 2 & 0 & $6(9)$ & 6 & 412 & 477 & $\mathbf{0 . 8 6}$ \\
\hline Mean & & & & & & & $38(/ \mathrm{p})$ & $58(/ \mathrm{p})$ & $0.67(/ \mathrm{p})$ \\
\hline \hline
\end{tabular}

Table 1. Accuracy and efficiency of the non-failure analysis (times in $\mathrm{mS}$ ).

Similarly, ACov shows two figures (both corresponding to the analysis presented in this paper): the number of predicates detected to cover all of their (calling) types (variants), and (between parenthesis), the number of predicates detected to cover some of their (calling) types. Cov is the number of predicates detected to cover their (calling) types by the analysis in [9].

$\mathbf{T}_{A F}$ and $\mathbf{T}_{F}$ are the total time (in milliseconds) required by the analysis presented in this paper and the analysis in [9] respectively (both of which include the time required to derive the modes and types). The timings were taken on a medium-loaded Pentium IV Xeon 2.0Ghz with two processors, 1Gb of RAM memory, running Red Hat Linux 8.0, and averaging several runs and eliminating the best and worst values. Ciao version 1.9.111 and CiaoPP-1.0 were used.

Analysis time averages (per predicate) are also provided in the last row of the table. From these numbers, it is clear that the new implementation based on the abstract interpretation engine is more efficient than the previous one. It is also more precise, as shown for example in the benchmarks $M v$, Zebra, Family, Blocks, Reach, and Plan. 


\section{Conclusions}

We have described a non-failure analysis based on abstract interpretation, which extends the previous proposal of Debray et al. Our analysis improves in precision, and enjoys a clear theoretical setting, and a simpler implementation. Also, the implementation is more efficient. The abstract domain underlying the analysis can be easily modified to cater for a determinacy analysis. Such an analysis, provided with a depth-k abstraction, would be the abstract interpretation counterpart of determinacy analyses such as that of [6]. We are currently working on the verification of this proposition.

The implemented analysis we have described in this paper is currently integrated in CiaoPP, and is being used for lower-bounds cost analysis, granularity control, and program debugging. Arguably, although our presentation covers strictly constraint logic programming, the technique could be easily applied to functional logic languages with similar results, as is indeed the case in the Ciao system, where the analysis presented works without modification for Ciao's functional subset or for combinations of functions and predicates.

\section{References}

1. C. Braem, B. Le Charlier, S. Modart, and P. Van Hentenryck. Cardinality analysis of prolog. In Proc. International Symposium on Logic Programming, pages 457471, Ithaca, NY, November 1994. MIT Press.

2. M. Bruynooghe. A Framework for the Abstract Interpretation of Logic Programs. Tech. Rep. CW62, Dept. of C.S., Katholieke Universiteit Leuven, October 1987.

3. M. Bruynooghe. A Practical Framework for the Abstract Interpretation of Logic Programs. Journal of Logic Programming, 10:91-124, 1991.

4. F. Bueno, P. López-García, G. Puebla, and M. Hermenegildo. The Ciao Prolog Preprocessor. Technical Report CLIP1/04, Technical University of Madrid (UPM), Facultad de Informática, 28660 Boadilla del Monte, Madrid, Spain, January 2004.

5. P. Cousot and R. Cousot. Abstract Interpretation: a Unified Lattice Model for Static Analysis of Programs by Construction or Approximation of Fixpoints. In ACM Symposium on Principles of Programming Languages, pages 238-252, 1977.

6. Steven Dawson, C. R. Ramakrishnan, I. V. Ramakrishnan, and R. C. Sekar. Extracting determinacy in logic programs. In David S. Warren, editor, Proceedings of the Tenth International Conference on Logic Programming, pages 424-438, Budapest, Hungary, 1993. The MIT Press. 
7. S. K. Debray and D. S. Warren. Automatic Mode Inference for Prolog Programs. Journal of Logic Programming, 5(3):207-229, September 1988.

8. S.K. Debray and N.-W. Lin. Static Estimation of Query Sizes in Horn Programs. In Third International Conference on Database Theory, Lecture Notes in Computer Science 470, pages 515-528, Paris, France, December 1990. Springer-Verlag.

9. S.K. Debray, P. López-García, and M. Hermenegildo. Non-Failure Analysis for Logic Programs. In 1997 International Conference on Logic Programming, pages 48-62, Cambridge, MA, June 1997. MIT Press, Cambridge, MA.

10. S.K. Debray, P. López-García, M. Hermenegildo, and N.-W. Lin. Lower Bound Cost Estimation for Logic Programs. In 1997 International Logic Programming Symposium, pages 291-305. MIT Press, Cambridge, MA, October 1997.

11. G. Gupta, E. Pontelli, K. Ali, M. Carlsson, and M. Hermenegildo. Parallel Execution of Prolog Programs: a Survey. ACM Transactions on Programming Languages and Systems, 23(4):472-602, July 2001.

12. M. Hermenegildo, F. Bueno, G. Puebla, and P. López-García. Program Analysis, Debugging and Optimization Using the Ciao System Preprocessor. In 1999 International Conference on Logic Programming, pages 52-66, Cambridge, MA, November 1999. MIT Press.

13. M. Hermenegildo, G. Puebla, F. Bueno, and P. López-García. Program Development Using Abstract Interpretation (and The Ciao System Preprocessor). In 10th International Static Analysis Symposium (SAS'03), number 2694 in LNCS, pages 127-152. Springer-Verlag, June 2003.

14. M. Hermenegildo, G. Puebla, K. Marriott, and P. Stuckey. Incremental Analysis of Constraint Logic Programs. ACM Transactions on Programming Languages and Systems, 22(2):187-223, March 2000.

15. N. Jones and H. Sondergaard. A semantics-based framework for the abstract interpretation of prolog. In Abstract Interpretation of Declarative Languages, chapter 6 , pages 124-142. Ellis-Horwood, 1987.

16. H. Mannila and E. Ukkonen. Flow Analysis of Prolog Programs. In Fourth IEEE Symposium on Logic Programming, pages 205-214, San Francisco, California, September 1987. IEEE Computer Society.

17. C.S. Mellish. Abstract Interpretation of Prolog Programs. In Third International Conference on Logic Programming, number 225 in LNCS, pages 463-475. SpringerVerlag, July 1986.

18. K. Muthukumar and M. Hermenegildo. Deriving A Fixpoint Computation Algorithm for Top-down Abstract Interpretation of Logic Programs. Technical Report ACT-DC-153-90, Microelectronics and Computer Technology Corporation (MCC), Austin, TX 78759, April 1990.

19. K. Muthukumar and M. Hermenegildo. Compile-time Derivation of Variable Dependency Using Abstract Interpretation. Journal of Logic Programming, 13(2/3):315-347, July 1992.

20. T. Sato and H. Tamaki. Enumeration of Success Patterns in Logic Programs. Theoretical Computer Science, 34:227-240, 1984.

21. A. Waern. An Implementation Technique for the Abstract Interpretation of Prolog. In Fifth International Conference and Symposium on Logic Programming, pages 700-710, Seattle, Washington, August 1988.

22. R. Warren, M. Hermenegildo, and S. K. Debray. On the Practicality of Global Flow Analysis of Logic Programs. In Fifth International Conference and Symposium on Logic Programming, pages 684-699. MIT Press, August 1988. 